\title{
Gradhiva
}

Revue d'anthropologie et d'histoire des arts

Matthieu Charle, Coeur d'Alene : ethnohistoire d'une communauté indienne d'Amérique du Nord, préface d'Emmanuel Désveaux

La Roche-sur-Yon, Dépaysage, coll. « L’entaille d'Orphée », 2019

\section{Benjamin Balloy}

\section{(2) OpenEdition}

\section{Journals}

Édition électronique

URL : http://journals.openedition.org/gradhiva/5232

DOI : $10.4000 /$ gradhiva.5232

ISSN : 1760-849X

Éditeur

Musée du quai Branly Jacques Chirac

Édition imprimée

Date de publication : 2 septembre 2020

Pagination : 174-175

ISBN : 978-2-35744-131-6

ISSN : 0764-8928

Référence électronique

Benjamin Balloy, « Matthieu Charle, Coeur d'Alene : ethnohistoire d'une communauté indienne d'Amérique du Nord, préface d'Emmanuel Désveaux », Gradhiva [En ligne], 31 | 2020, mis en ligne le 31 mars 2021, consulté le 01 avril 2021. URL : http://journals.openedition.org/gradhiva/5232 ; DOI : https://doi.org/ 10.4000/gradhiva.5232

Ce document a été généré automatiquement le 1 avril 2021.

(C) musée du quai Branly 
Matthieu Charle, Coeur d'Alene: ethnohistoire d'une communauté indienne d'Amérique du Nord, préface d'Emmanuel Désveaux

La Roche-sur-Yon, Dépaysage, coll. «L'entaille d'Orphée », 2019

Benjamin Balloy

\section{RÉFÉRENCE}

Matthieu Charle, Coeur d'Alene : ethnohistoire d'une communauté indienne d'Amérique du Nord, préface d'Emmanuel Désveaux, La Roche-sur-Yon, Dépaysage, coll. « L'entaille d'Orphée », 2019, 317 p. 


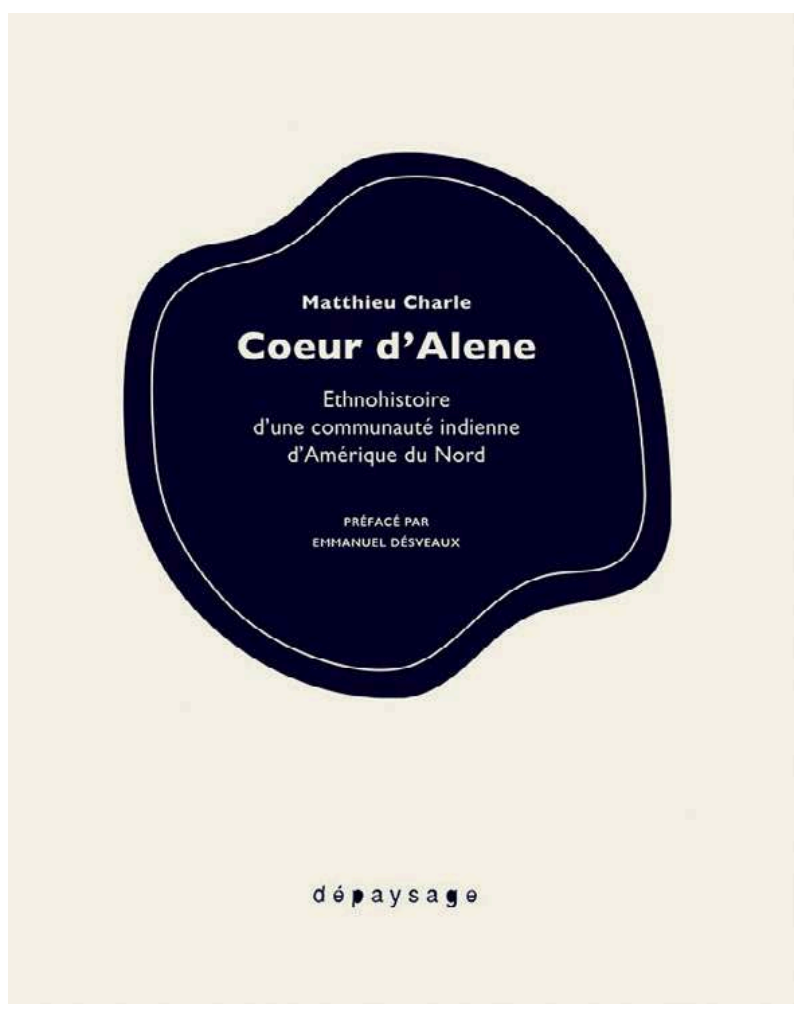

Le terrain nord-américain a peu séduit les ethnographes formés en France. La publication d'une monographie, basée sur la longue enquête de Matthieu Charle, entre 2002 et 2005, au sein de la réserve des Coeur d'Alene ${ }^{1}$, est à ce titre remarquable. Sans s'appesantir, l'auteur réussit à nous introduire aux décourageantes difficultés de l'observation participante dans une communauté amérindienne contemporaine. Comme l'indique le sous-titre, sa démarche relève plus exactement d'une " ethnohistoire », au sens où Raymond DeMallie notamment l'a définie, afin "d'écrire un récit et d'élaborer une ethnographie historique les plus profondément ancrés dans la culture d'origine » (p. 40). Le livre est rédigé dans une prose claire et agréable, écrit à la première personne et mêle étroitement les registres analytique, réflexif et théorique. Par son propos, il vise à «identifier puis analyser les moyens par lesquels la communauté Coeur d'Alene a négocié la pérennité d'éléments sociaux fondamentaux à sa reproduction avec un environnement changeant, depuis le milieu du xix siècle jusqu'à nos jours» (p.40). Les Coeur d'Alene appartiennent au groupe des communautés amérindiennes de langue salish du Plateau, vaste région de transition biogéographique entre les Plaines et les Rocheuses à l'est, les chaînes côtières des Cascades à l'ouest, et les plateaux désertiques au sud. Leur territoire traditionnel - sur lequel la réserve, dans l'actuel état de l'Idaho, est en partie établie - était traversé par différents affluents du fleuve Spokane et centré en particulier sur le lac Coeur d'Alene.

L'ouvrage commence par aborder, dans ses grandes lignes, l'ethnographie classique des Coeur d'Alene historiques (partie I), c'est-à-dire ceux dont l'entreprise de «sauvetage mémoriel ", effectuée par James Teit à l'instigation de Franz Boas, au tournant du $\mathrm{xx}^{\mathrm{e}}$ siècle, a fixé la trace - comme c'est le cas de presque toutes les sociétés du Plateau. L'ethnographie de cette région, considérée comme très lacunaire, pâtit du faible recul historique qu'offre la documentation et de la rapidité des transformations que tous ces groupes connaissent dans la deuxième moitié du XIX siècle. L'approche culturaliste, que récuse vigoureusement Charle, est toujours très prégnante dans l'anthropologie du 
sous-continent. Dans le schéma des aires culturelles, le "Plateau ", tel que caractérisé par Verne Ray dans les années 1940, semble surtout souffrir d'une identité en creux, appréhendée "par défaut» (p.290). Leurs attributs culturels et sociaux sont supposément illustratifs d'un état transitoire entre les sociétés, mieux connues, de la côte Nord-Ouest et des Plaines.

Dans la deuxième partie, l'auteur expose chronologiquement la transition menant les Coeur d'Alene du choc épidémique et démographique de la fin du xviII ${ }^{e}$ siècle jusqu'aux années 1960, à l'arrêt des politiques fédérales d'assimilation. L'installation des missionnaires jésuites sur leur territoire vers 1850 et les différentes transformations du mode de vie - dont la plus visible est l'adoption massive de l'agriculture mécanisée - et de l'organisation sociale font l'objet d'un exposé attentif. La troisième partie présente la thèse principale de l'auteur: le rôle fondamental qu'a joué la mission dans la recomposition des rapports structurant entre familles étendues, au sein de la communauté, ainsi qu'entre ses membres et le monde extérieur. C'est ici que les données ethnographiques sont principalement convoquées. À l'époque contemporaine, dans la diversité de récits consacrés par des traditions familiales opposées voire concurrentes, le souvenir - contrasté et contesté - du rôle de la mission jésuite a finalement servi à la fois à reconfigurer le "jeu interactionnel» au sein de la communauté et à alimenter une forme de continuation de la "politique tribale de consensus, qui a permis au collectif Coeur d'Alene, jusqu'aujourd'hui, de faire face à des bouleversements majeurs sans pour autant remettre en cause son ontologie» (p. 131). Au centre de cette querelle du souvenir du rôle de la mission : la langue Coeur d'Alene. Et ce paradoxe qui incite l'auteur à adopter une perspective ethnohistorique, qui ne sépare pas l'étude des documents de celle des récits et mémoires transmis, fussent-ils contradictoires : tandis que le souvenir critique du rôle de la mission tend, depuis les années 1960, à se cristalliser autour de l'affirmation que la pratique de la langue Coeur d'Alene a été interdite par les missionnaires, les derniers locuteurs âgés qu'il rencontre se trouvent justement appartenir au groupe des familles éduquées dans le cadre de la mission et restés proches de celle-ci. La quatrième partie, à partir de la notion de communauté, explore la question de la cohésion sociale telle qu'elle a été et est, aujourd'hui encore, renégociée et réaffirmée. La cinquième partie ("adaptation, résistance ou mimétisme») revient sur des questions soulevées dans les pages précédentes. Le cas des Coeur d'Alene contemporains est mis en vis-à-vis d'un choix de textes d'anthropologues ou d'ethnohistoriens, afin, dit l'auteur, d'«affiner la réflexion " (p. 230). La coda et la brève conclusion centrées sur le récit d'une anecdote personnelle demeurent conformes à l'esprit du livre.

4 Une des indéniables réussites de ce texte est d'éviter l'écueil d'une analyse du présent uniquement à l'aune des éventuelles «survivances» ou traces d'un passé ethnographique - bon grain culturel à séparer de l'ivraie d'une contemporanéité sociologique acculturante. C'est au contraire l'interaction entre un passé commun - une ethnographie et une histoire tribale partagées - et les négociations différenciées successives de cette histoire par divers segments familiaux qui offre, l'auteur nous en convainc, le point de vue le plus pertinent pour saisir à la fois les continuités et les ruptures, les oublis et les réappropriations au sein de la communauté Coeur d'Alene. Un autre aspect remarquable du travail de Charle est de prendre pour objet de son enquête cette tension structurante (dont la langue est l'enjeu le plus manifeste et le plus contradictoire) et certaines de ses expressions, au lieu d'adhérer à la définition de façade d'une communauté politique autochtone nécessairement uniforme et unifiée. 
Par la mise en évidence des différents modes d'insertion des Coeur d'Alene dans l'Amérique contemporaine, décrire leur "culture» revient à rendre manifeste la pluralité des manières d'être Coeur d'Alene - et à comprendre ce que cet être veut dire. Différentes études de cas permettent de souligner la complexité des rapports en jeu. Certaines pratiques s'inscrivent apparemment dans une forme de continuité culturelle et historique - de l'importance de la trame des relations de parenté, pour saisir la structure des interactions signifiantes, à la passion pour les jeux de hasard du type Stick Game, en passant par une éventuelle survivance de la valorisation de l'ethos guerrier via l'attention portée aux vétérans. D'autres manifestations sont plus longuement analysées par l'auteur: par exemple, les formes locales de développement des powwow; ou bien, le relatif désintérêt pour l'apprentissage et la transmission de la langue Coeur d'Alene, même si la tribu s'est dotée d'un centre linguistique très actif. Au sein de la communauté, certains membres choisissent ou non de s'engager dans des pratiques, se les approprient pour les investir d'un jeu polysémique d'intentions ou, au contraire, ne les considèrent pas comme allant de soi pour se sentir pleinement membres de la communauté. Charle y voit autant de symptômes de cette "renégociation » de la cohésion sociale. On regrette toutefois que l'ethnographie, notamment du pow-wow et des jeux, ne soit pas plus détaillée. Cela aurait sans doute permis d'approfondir l'enquête sociologique sur les segments familiaux qui participent du factionnalisme latent dans la communauté Coeur d'Alene - un factionnalisme qui n'est d'ailleurs jamais véritablement défini ou précisé.

$5 \quad$ Ajoutons que le choix de n'aborder la discussion théorique que dans les deux dernières parties du livre ne nous a pas semblé heureux, quand bien même il s'agirait là d'épouser le mouvement de la recherche en train de se faire (p. 42) - "dans l'atelier ", disait Marc Bloch. En effet, le lecteur aurait gagné à trouver l'exposé des cas et leur analyse mêlés, sans avoir par ailleurs le sentiment d'une synthèse éludée.

Outre la grande qualité de l'édition du livre, publié par une toute jeune maison vendéenne formulant le souhait de se consacrer «à la connaissance des peuples autochtones d'hier et d'aujourd'hui ", on notera la stimulante préface d'Emmanuel Désveaux. Ceux qu'une perspective structuraliste à l'échelle de l'Amérique du Nord ne laisse pas indifférents y trouveront des pistes de recherche originales pour dépasser l'approche culturaliste de l'ethnologie des sociétés du Plateau.

\section{NOTES}

1. Comme l'auteur s'en explique, cette graphie correspond à celle adoptée par les Coeur d'Alene eux-mêmes. 


\section{AUTEURS}

BENJAMIN BALLOY

benjamin.balloy[at]quaibranly.fr 